\title{
Enhancing spatial resolution in digital holographic microscopy by biprism structured illumination
}

\author{
Emilio Sánchez-Ortiga, ${ }^{1, *}$ Manuel Martínez-Corral, ${ }^{1}$ Genaro Saavedra, ${ }^{1}$ and Jorge Garcia-Sucerquia ${ }^{1,2,3}$ \\ ${ }^{1} 3 D$ Imaging and Display Laboratory, Department of Optics, University of Valencia, E-46100 Burjassot, Spain \\ ${ }^{2}$ Universidad Nacional de Colombia Sede Medellin, School of Physics, A.A: 3840-Medellin-050034-Colombia \\ ${ }^{3}$ e-mail: jigarcia@unal.edu.co \\ *Corresponding author: emilio.sanchez@uv.es
}

Received October 3, 2013; revised February 24, 2014; accepted March 7, 2014; posted March 7, 2014 (Doc. ID 198890); published March 27, 2014

\begin{abstract}
A novel and efficient architecture of a structured-illumination digital holographic microscope (DHM) is presented. As the DHM operates at the diffraction limit, its spatial resolution on label-free imaging of transparent samples is improved by illuminating the sample with a structured illumination produced by a Fresnel's biprism. The theoretical analysis of the method forecasts a twofold improvement of the spatial resolution. The proposed method requires only two images to improve the spatial resolution, which eases the process of unmixing the high-resolution components by means of an unknown phase-shift procedure. Numerical modeling and experimental results validate the theoretical findings. (c) 2014 Optical Society of America

OCIS codes: (090.1995) Digital holography; (110.0180) Microscopy; (100.6640) Superresolution; (260.1960) Diffraction theory.

http://dx.doi.org/10.1364/OL.39.002086
\end{abstract}

Label-free quantitative phase microscopy (QPM) and numerical refocusing are particularities that make digital holographic microscopy (DHM) an interesting tool for life and material sciences. Both features are supported on the optical recording of digital holograms and their numerical a-posteriori reconstruction to retrieve the complex optical field scattered by the sample. This process leads to a microscopy tool where the spatial resolution is determined by the perfect marriage between the optical and the numerical processing of the optical information scattered by the specimen. Therefore, in the best possible scenario where the DHM operates at diffraction limit [1] , its spatial resolution equals that of the optical system utilized for the recording of the digital hologram. Therefore, DHM can distinguish two particles placed $\Delta r$ apart if $\Delta r \geq \lambda / 2$ NA [2,3] with $\lambda$ the illumination wavelength and NA the numerical aperture of the imaging system. To increase the resolving power either shorter wavelengths can be utilized [4] or the NA can be increased [1,5-7]. The enlargement of the NA, namely, the increasing of the capability of gathering spatial frequencies, can be achieved either in the space [5] or in the spatial frequency-domain $[1,7,8]$. The latter methods can be categorized as those that synthesize an enlarged transfer function. Perhaps the most notable architecture that improves the spatial resolution by this way is the structured illumination microscopy (SIM) $[9,10]$. The methodology of SIM has been recently adopted in DHM to improve the spatial resolution as transparent samples are imaged $[\underline{8}, \underline{11}]$. The needed multiple images to synthesize the extended transfer functions are obtained either by using a spatial light modulator [8] or by the interference of two diffracted waves from a diffraction grating [11]. In this Letter, we also present the enhancement of the spatial resolution in DHM by using a SIM-like methodology. The main differences with the recently reported advances in this direction $[\underline{8}, \underline{11}]$ are: (1) the use of a Fresnelś biprism [2] to produce periodic illumination pattern over the object plane; (2) the use of unknown phase-shifting to synthesize the enlarged transfer function; and (3) the optimized utilization of the space bandwidth of the recording device.

DHM takes advantage of the interferometic recording of a reference $R(\mathbf{x})$ with a propagated object wave $O_{z}^{\prime}(\mathbf{x})$ to encode the phase information that this latter wave carries. In particular when the DHM operates in afocal-telecentric mode [12], as it is the case in this work, the spectrum of the recorded intensity does not vary with the recording distance. The intensity recorded by the digital camera is therefore given by

$$
I(\mathbf{x})=\left|O_{z}^{\prime}(\mathbf{x})\right|^{2}+|R(\mathbf{x})|^{2}+O_{z}^{\prime *}(\mathbf{x}) R(\mathbf{x})+O_{z}^{\prime}(\mathbf{x}) R^{*}(\mathbf{x}),
$$

where $\mathbf{x}=(x, y), *$ means complex conjugated, and $O_{z}^{\prime}(\mathbf{x})=O^{\prime}(\mathbf{x}) \otimes \exp [i 2 \pi z / \lambda] \exp [i \pi / \lambda z] / i \lambda z$ is the propagated wave field from the image of the microscope to the recording plane. SIM-like strategies illuminate the sample with a periodic wave, field to improve the lateral resolution [8-11]. As illustrated in Fig. 1, in this proposal such periodic pattern is produced by illuminating a Fresnel's biprism with a plane wave, which creates two plane waves traveling with an angle that depends on the biprism angle and its index of refraction. On considering that the original plane wave comes from the collimation of a

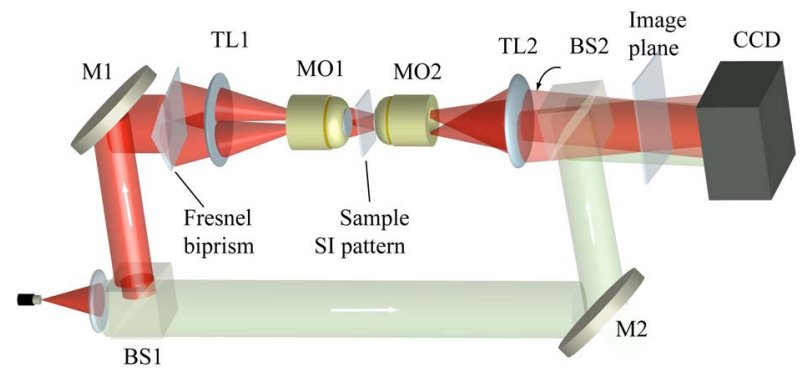

Fig. 1. Schematic illustration of the biprism structured illumination DHM (SI-DHM). 
point source, the Fresnel's biprism produces two mutually coherent virtual point sources located in the same plane as the point source and symmetrically displaced over such plane. The virtual point sources are imaged by the tube lens 1 (TL1) onto the entrance pupil of the illumination microscope objective (MO1), which produces two plane waves that interfere over the plane of the sample. The use of the biprism improves the light throughput, allows easy variation of the spatial frequency and orientation of the periodic illumination, and reduces the complexity of the optical set-up. As the sample is illuminated by the periodic wave field produced by the Fresnel biprism, the complex optical field in the object plane of the imaging microscope objective (MO2), assuming that its NA is equal to the one for MO1, can be written as

$$
O^{\prime}(\mathbf{x})=\left\{O(\mathbf{x} / M) \cos \left(2 \pi \mathbf{f} \cdot \mathbf{x} / M+\left(\phi_{j}+\phi_{0}\right)\right)\right\} \bigotimes_{2} h(\mathbf{x}) .
$$

In Eq. (2) some constants have been dropped, being $\otimes_{2}$ the $2 \mathrm{D}$ convolution operator, $\mathbf{f}$ accounts for the spatial frequency and orientation of the periodic wave field, $h(\mathbf{x})$ and $M$ the amplitude point spread function and lateral magnification of the MO2, respectively, $\phi_{j}$ a random phase-shift such that $0<\phi_{j}<\pi$, and $\phi_{0}$ the initial phase of the periodic wave field with respect to the phaseshift $\phi_{j}$.

Assuming the reference wave to be a plane wave that interferes with the propagated object wave creating a carrier frequency given by the wave vector $\alpha$, the Fourier transform of the recorded hologram can be written as

$$
\tilde{I}(\mathbf{k})=\mathrm{DC}(\mathbf{k})+\tilde{O}_{z}^{\prime *}(\mathbf{k}) \otimes \delta(\mathbf{k}-\alpha)+\tilde{O}_{z}^{\prime}(\mathbf{k}) \otimes \delta(\mathbf{k}+\alpha),
$$

where three orders can be distinguished: the DC order, placed at the origin of spatial-frequency coordinates, and two symmetrically displaced orders, so-called +1 and -1 , with a separating distance controlled on the angle of the reference wave vector $\boldsymbol{\alpha}$. The proper selection of the wave vector $\boldsymbol{\alpha}$ allows us the optimized used of the space bandwidth such that the three orders are perfectly isolated and occupied fully in the available space $[\underline{1}, \underline{13}]$. Under this condition, the so-called +1 order can be filtered out and then relocated to the center of the spatial-frequency coordinates for compensating the reference wave vector $\boldsymbol{\alpha}$.

In the angular spectrum regime $z \rightarrow 0$, then the compensated complex wave field, which corresponds to the reconstructed object wave, is given by

$$
\begin{aligned}
\tilde{U}_{j}^{R}(\mathbf{k})= & \frac{1}{2}\left\{\tilde { O } ( M \mathbf { k } ) \otimes \left(\exp \left[i\left(\phi_{j}+\phi_{0}\right)\right] \delta(\mathbf{k}-M \mathbf{f})\right.\right. \\
& \left.\left.+\exp \left[-i\left(\phi_{j}+\phi_{0}\right)\right] \delta(\mathbf{k}+M \mathbf{f})\right)\right\} H(\mathbf{k}),
\end{aligned}
$$

where $H(\mathbf{k})$ is the coherent transfer function (CTF) of the $\mathrm{MO}$. As we consider a MO with a clear-circular pupil, the CTF is a bi-valuated circle transmitting the spatialfrequencies contained inside its support from the objectto the image-space. In Eq. (4), there are two mixed components within the CTF support. These components are symmetrically placed over the center of coordinates of the spatial-frequency domain at a distance depending on the carrier frequency generated by the Fresnel biprism f. These components bear complementary information of the spatial-frequencies contained in the object complex amplitude $[\underline{9}, \underline{10}]$. To separate these components, there is the need for two linearly independent equations that can be obtained by varying the phase-shift $\phi_{j}$. These phase-shifts are obtained by a lateral displacement of the Fresnel biprism. Therefore, two images $I_{1}$ and $I_{2}$, with different values of $\phi_{j}$ must be acquired; for instance, $\phi_{1}=0, \phi_{2}=\phi$. By solving the system of equations, we obtain the solutions:

$$
\begin{aligned}
& F_{1}(\mathbf{k})=\left(\tilde{U}_{1}^{R}(\mathbf{k}) \exp \left[-j\left(\phi / 2-\phi_{0}\right)\right]-\tilde{U}_{2}^{R}(\mathbf{k})\right) / \cos (\phi / 2) \\
& F_{2}(\mathbf{k})=\left(\tilde{U}_{2}^{R}(\mathbf{k}) \exp \left[j\left(\phi / 2+\phi_{0}\right)\right]-\tilde{U}_{1}^{R}(\mathbf{k})\right) / \cos (\phi / 2) .
\end{aligned}
$$

The phase-shift $\phi_{j}$ must be such that introduces at least a difference of 1 pixel between the two images, $I_{1}$ and $I_{2}$. After separating the components, they are properly weighted to compensate common zones and relocated to its correct position for synthesizing the enlarged CTF:

$$
F(\mathbf{k})=F_{1}^{W} \otimes \delta(\mathbf{k}+M \mathbf{f})+F_{2}^{W} \otimes \delta(\mathbf{k}-M \mathbf{f}) .
$$

To have an even enlargement of the CTF, the same procedure has to be applied to multiple directions on the spatial-frequency domain. The rotations of the periodic wave field over the object plane are obtained by simple rotating the Fresnel biprism. Therefore Eq. (6) can be extended for a number $S$ of rotated angles:

$$
F(\mathbf{k})=\sum_{s=1}^{S} F_{1, s}^{W} \otimes \delta\left(\mathbf{k}+M \mathbf{f}_{s}\right)+F_{2, s}^{W} \otimes \delta\left(\mathbf{k}-M \mathbf{f}_{s}\right),
$$

where $F_{j, s}^{W}$ are the average-weighted components. By performing the Fourier transform of the composed spectrum represented in Eq. (7), we obtain the object complex amplitude distribution spectrum with extended bandwidth. The fact that the spectrum does not vary with the propagation distance for the afocal telecentric DHM allows the composition of the enlarged CTF in any plane.

If we obtain the corresponding solutions $F_{1, s}^{W}, F_{2, s}^{W}$, then it is possible to see that the final composition of the spectrum can be expressed as

$$
F(\mathbf{k})=\tilde{O}(M \mathbf{k}) \sum_{s=1}^{S}\left(H\left(\mathbf{k}+M \mathbf{f}_{s}\right)+H\left(\mathbf{k}-M \mathbf{f}_{s}\right)\right) .
$$

The above equation shows that our system has a new synthetic CTF composed by $S$ pairs of displaced conventional CTFs; that is,

$$
H_{\text {synth }}(\mathbf{k})=\sum_{s=1}^{S}\left\{H\left(\mathbf{k}+M \mathbf{f}_{s}\right)+H\left(\mathbf{k}-M \mathbf{f}_{s}\right)\right\} .
$$

For the case in which $M \mathbf{f}$ corresponds to the cut-off frequency of the imaging system, namely $\mathbf{k}_{0}$, the synthetic 
CTF doubles in extension the CTF of the imaging system, hence the spatial resolution of the SI-DHM doubles that of the regular DHM. It is remarkable that through this composition performed at the diffraction limit of the imaging system, the complex optical of the object field remains flawless. Therefore, the method can be applied both to amplitude and phase-contrast resolution improvement.

A postprocessing algorithm is employed for retrieving the enhanced-resolution images. First, the algorithm crops the order +1 of each hologram with a circle of the size of the CTF. The position of the SI orders is detected by using peak detection. This provides also the relative intensity, $\sigma$, of the SI orders. Then the filtered digital holograms are combined, as stated in Eq. (5), in order to find the solutions $F_{1}$ and $F_{2}$, being $\phi$ unknown. For retrieving the value of $\phi$, we employ the following procedure, first we introduce a random value $\phi^{\prime} \in(0, \pi)$. Then in an iterative process, the value of $\phi^{\prime}$ is tuned for maximizing the value of $\sigma$. Ideally, $\sigma \rightarrow \infty$ makes sure that $\phi^{\prime}=\phi$, and Eq. (5) gives the exact solution of the system. In practice we have used a tolerance of $10^{-6}$ for the value of $\phi$. For locating the components at their correct position, we employ the values of the peak detection.

Our proposed method has been initially validated by means of numerical simulation of QPM. We modeled numerically the digital hologram acquisition with a diffraction limited system using as a sample a phase-modulated USAF 1951 test target with phase values of $\pi / 2$ for the lines and 0 for the background. The regular and synthetic CTFs are shown in Figs. 2(a) and 2(b), respectively. Then we reconstructed the quantitative phase images by regular DHM [Fig. 2(c)] and by SI-DHM [Fig. 2(d)]. As seen in these panels, the spatial resolution of the reconstructed image with SI-DHM is notably enhanced. Since we have computed a dimensionless simulation, the resolution enhancement is measured by the ratio of the values of $d$ for panels (c) and (d). While in panel (c), the smallest resolved element is the element 3 group 0 , in panel (d) is the element 3 group 1, which leads to a resolution ratio of two, as it was expected.

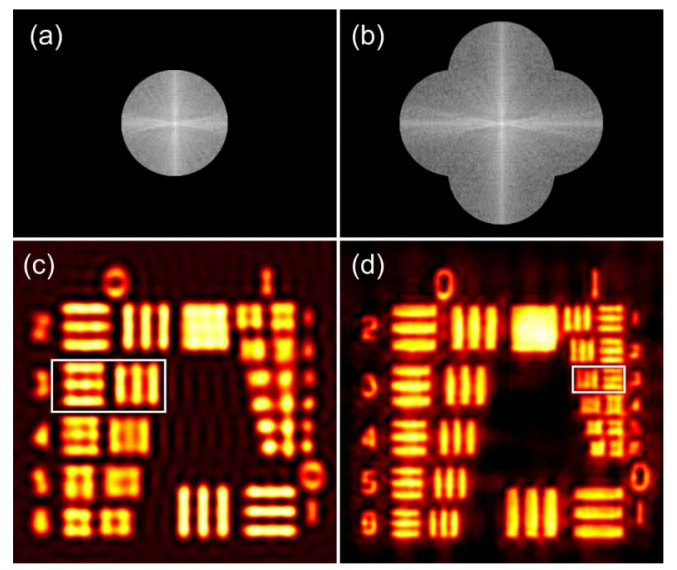

Fig. 2. Numerical modeling for testing the resolution enhancement in QPM via SI-DHM. Panels (a) and (b) are the CTFs for regular- and SI-DHM, respectively, whereas (c) and (d) are the corresponding quantitative phase images. The white rectangles show the highest resolved elements.
The SI-DHM was implemented on a regular DHM configuration based on a Mach-Zehnder interfometer in which an He-Ne laser was utilized as an optical source, as shown in Fig. 1. The concept was experimentally proved by imaging an USAF 1951 test target with a low-NA MO2 $(2 \times \mathrm{NA}=0.1)$. All the tube lenses used in our experiment have a focal length of $200 \mathrm{~mm}$. The holograms were recorded on a CCD camera with $1224 \times$ 1024 square pixels of $6.9 \mu \mathrm{m}$ pixel pitch. The structured illumination in the object plane was generated by a Fresnel biprism with 5 deg angle and index of refraction 1.54. With the biprism in place, we record a first hologram. The biprism is laterally displaced for introducing a random phase-shift $\phi_{j}$ to record the second hologram. With these two holograms, we have synthesized the CTF. To show in just one image the enhancement on the spatial resolution with SI-DHM, the CTF has been enlarged along one direction while the orthogonal is left unmodified. Figure 3(a) shows the synthetic CTF.

Such one-direction enlargement renders spatial resolution of the order of $71.8 \mathrm{lp} / \mathrm{mm}(40.3 \mathrm{lp} / \mathrm{mm})$ for the horizontal (vertical) lines of the USAF test target. Panel (b) shows the amplitude contrast reconstructed image with two-time zoomed-in areas for better representation of the achieved.

The SI-DHM proposed has been also applied on imaging a label-free biological sample. A section of the head of a drosophila melanogaster fly was imaged with an MO $50 \times \mathrm{NA}=0.55$. For this experiment, the same biprism as before was used. In that case, for producing the desired spatial frequency of the pattern, we used a projection $\mathrm{MO}$ with $\mathrm{NA}=0.55$. We have rotated sequentially the biprism for producing two barely orthogonal patterns. In this case, the synthetic CTF is constructed following Eq. (7). In Fig. 4 are illustrated the CTFs for regular DHM [panel (a)] and SI-DHM [panel (b)]. The clearly enlarged synthetic CFT for SI-DHM extends the cut-off frequency of the imaging system on both amplitude and phase-contrast imaging. The amplitudecontrast images for the studied specimen for regular DHM [Fig. 4(c)] and SI-DHM [Fig. 4(d)] are shown. The zoomed-in areas (1.5 times) bounded by the green circles, show the enhancement of the spatial resolution for the SI-DHM. The spike-like antenna, barely visible in panel (c), is seen with great detail in panel (d).

From the same reconstructed complex amplitudes utilized in Fig. 4, the phase-contrast images were calculated. The Fig. $\underline{5}$ panel (a)/(b) shows the reconstructed phase

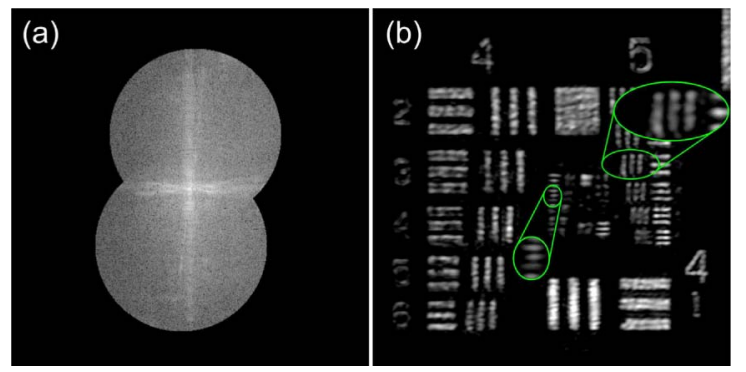

Fig. 3. Experimental one-direction SI-DHM. Panel (a) shows the one direction synthetic CTF. Panel (b) is the contrastamplitude reconstructed image. The colored ellipses remark the smallest resolved elements along each direction. 

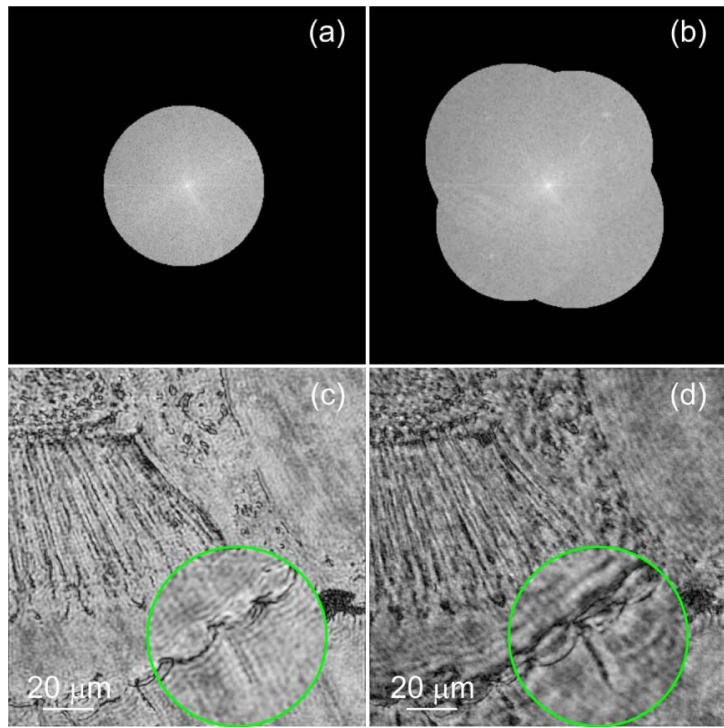

Fig. 4. Regular DHM versus SI-DHM, amplitude. Panel (a)/(b) shows the CTF for regular DHM/SI-DHM. Panel (c)/(d) is the amplitude-contrast image of a section of the head of a drosophila melanogaster fly for regular DHM/SI-DHM.

image for the regular DHM/SI-DHM. The encircled areas show phase information visible clearly in panel (b) whereas it is blurred in panel (a). The areas surrounded by the green rectangles at the bottom were 1.5 times zoomed-in in the upper-right corners of the respective panels. The spike-like antenna and the white tissue with black porous, which are perfectly visible with SI-DHM, are totally blurred in the regular DHM.

In summary, we have presented a method to improve the spatial resolution in DHM operating in both amplitude- and phase-contrast imaging. The introduction of the biprism renders into a more light-efficient and simpler SI-DHM than those recently introduced by other authors. Additionally, the biprism allows the phase-shifting/ rotation of the pattern by displacing/rotating it. The use of coherent light reduces the needed images to synthesize the enlarged CTFs; only two images per direction are necessary to achieve the forecasted twofold improvement of the spatial resolution. The synthesizing method of the CTF only requires an unknown phase-shift that can be extracted from the solutions of a system of two equations, easing the implementation of the SI-DHM with respect to other applications of SI that require, at least, three images per direction.

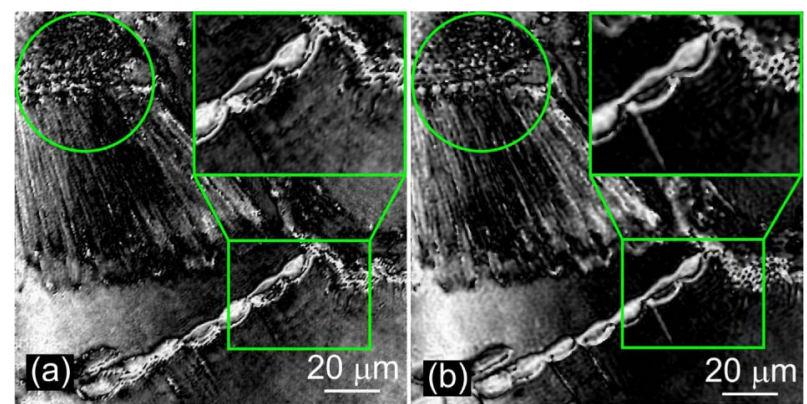

Fig. 5. Regular DHM versus SI-DHM, phase. Phase-contrast images of a section of the head of a drosophila melanogaster fly for regular DHM (a) and SI-DHM (b).

This work was supported in part by the Ministerio de Ciencia e Innovación, Spain (grant DPI2012-32994) and also by Generalitat Valenciana (grant PROMETEO2009077). J. Garcia-Sucerquia gratefully acknowledges the Visiting Scholar Fellowship from the Universidad de Valencia.

\section{References}

1. Y. Cotte, F. Toy, P. Jourdain, N. Pavillon, D. Boss, P. Magistretti, P. Marquet, and C. Depeursinge, Nat. Photonics 7, 113 (2013).

2. M. Born and E. Wolf, Principles of Optics, 7th ed. (Cambridge University, 2005).

3. E. Cuche, F. Bevilacqua, and C. Depeursinge, Opt. Lett. 24, 291 (1999).

4. A. Faridian, D. Hopp, G. Pedrini, U. Eigenthaler, M. Hirscher, and W. Osten, Opt. Express 18, 14159 (2010).

5. J. Garcia-Sucerquia, W. Xu, M. H. Jericho, and H. J. Kreuzer, Opt. Lett. 31, 1211 (2006).

6. C. Yuan, G. Situ, G. Pedrini, J. Ma, and W. Osten, Appl. Opt. 50, B6 (2011).

7. V. Mico, Z. Zalevsky, C. Ferreira, and J. García, Opt. Express 16, 19260 (2008).

8. P. Gao, G. Pedrini, and W. Osten, Opt. Lett. 38, 1328 (2013).

9. M. G. L. Gustafsson, J. Microsc. 198, 82 (2000).

10. M. G. L. Gustafsson, Proc. Natl. Acad. Sci. USA 102, 13081 (2005).

11. S. Chowdhury and J. Izatt, Biomed. Opt. Express 4, 1795 (2013).

12. A. Doblas, E. Sánchez-Ortiga, M. Martínez-Corral, G. Saavedra, P. Andrés, and J. Garcia-Sucerquia, Opt. Lett. 38, 1352 (2013).

13. E. Cuche, P. Marquet, and C. Depeursinge, Appl. Opt. 39, 4070 (2000). 